\title{
Balancing of hybriid assembly systems using a simulation approach
}

Gert Zülch, Thorsten Vollstedt and Reinhard Müller

ifab-Institute of Human and Industrial Engineering, (Institut fïr Arbeitswissenschaft und Betriebsorganisation), University of Karlsruhe (TH), Kaiserstrasse 12, D - 76128 Karlsruhe, Germany.Email:Thorsten.Vollstedt@mach.uni-karlsruhe.de

\begin{abstract}
The short-lived nature of products requires production enterprises to plan new production systems in ever shorter intervals. The enterprises are thus prompted to improve their planning processes and in particular to shorten their planning times. Computer supported planning tools can be used to develop planning alternatives and to examine them, using simulation, with respect to their dynamic attributes. A substantial problem can be seen in the conception of a hybrid assembly system containing a similar amount of automated and manual stations. The procedure PAMELA was developed at the ifab-Institute of Human and Industrial Engineering at the University of Karlsruhe for the design of suitable planning solutions for hybrid assembly systems (Muller 2002; Zulch, Muller 2001a). This procedure is based on the description of work content and the dependencies of assembly operations as well as several layered precedence diagrams. Simulation is called upon for the assessment and choice of alternative planning solutions. The use of the procedure PAMELA will be demonstrated in this article using an application example.
\end{abstract}

Keywords

Hybrid Assembly System, Simulation, Balancing of Assembly Systems.

\section{MODELLING HYBRID ASSEMBLY TASIKS}

When planning work sequences in assembly systems, personnel are generally the resource which creates bottlenecks and which determines the efficiency of the system. However, if a hybrid system in which assembly processes are performed manually as well as automatically is planned, an exclusive consideration of human resources is not sufficient. In hybrid assembly tasks all process participants must be considered simultaneously. For this purpose, in addition to personnel as a process participant, the machinery resources and the work object itself must also be regarded explicitly in the configuration of the assembly sequence. From the point of view of these process participants, in particular the assembly sequence can be represented in various ways (Zulch, Muller 2001b). When executing the assembly operations these process participants can be bound for varying lengths of time. 
For example, in the assembly of gearing mechanisms, certain parts for several variants must be warmed up directly prior to assembly. This requires a certain period of time. After these parts have been assembled the work object must cool down before the subsequent assembly operation can be carried out. When configuring assembly sequences these work-object-based time periods must be taken into account in such a way that the personnel or the machinery resources are available for other operation.

A central element in the new procedure PAMELA (Planung automatisierter und manueller Montagen mit Leistungsabstimmung) is the modelling of the hybrid assembly tasks from the point of view of the worker, the machinery resource and the work object. For this purpose an assembly precedence diagram, which describes the temporal-logistic dependencies in the product assembly in a network manner, is used (cf. Prenting, Battaglin, 1964; Zulch, Muller 2001b). As is generally known, each node in the precedence diagram is equivalent to an assembly operation. The procedure PAMELA differentiates, however between manual operations, in which the worker is in the foreground, automated operations, which can only be carried out with help from machinery resources, as well as work-object-orientated operations, which are defined by an operation without intervention of a person or a machine (e.g. drying or curing operations).

Figure 1 is an example of a hybrid assembly task consisting of four operations represented as a precedence diagram, resulting in a sequence of assembly operations.

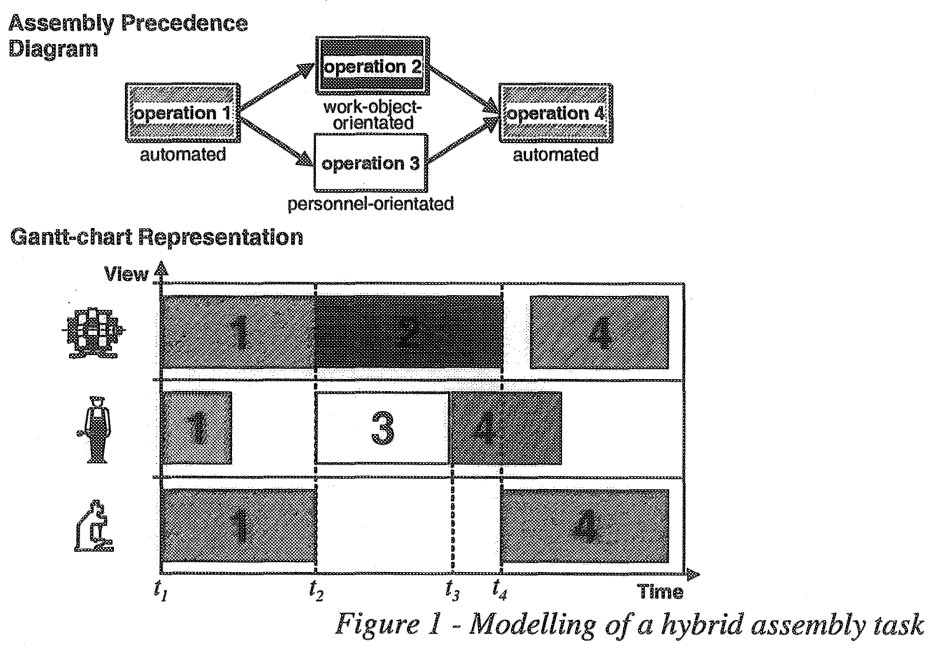

Assembly operation 1 is automated and is characterized by a short manual activity time. Assembly operation 2 is a work-object-orientated operation in which no time allotment is needed either for personnel or for machinery resources. Assembly operation 3 is worker-orientated and the work content comprises preassembly of components, for which the main work-object is not required as a basic part so that no related operation time is incurred. When considering the precedence restrictions, the assembly operations 2 and $3 \mathrm{can}$, in this example, be planned to be executed in parallel. With classical planning approaches these operations would 
have had to be performed successively. Furthermore, preparation activities for assembly operation 4 can already begin before operation 2 has been completed.

\section{DEVELOPMENT OF THE ASSEMBLY SYSTEM}

After the generation of the precedence diagram with its different views of the process participants, the assembly system is then to be developed while taking the precedence diagram into consideration. This includes the organizational linkage of assembly tasks, workers and machinery resources in the hybrid assembly system to be planned. For this purpose a rough structuring of the assembly system must be carried out in order to divide the assembly tasks into more or less decoupled subsystems, such as for example pre--, intermediate- and final-assembly. Finally, a suitable form of capacity partitioning must be defined and, within the framework of a capacity balancing, an even distribution of the work content to the individual work stations undertaken. Therefore, a planning algorithm can be used. The general form of this algorithm is shown in Figure 2. An example of the technical and personnel structures of an assembly system is represented in Figure 3.

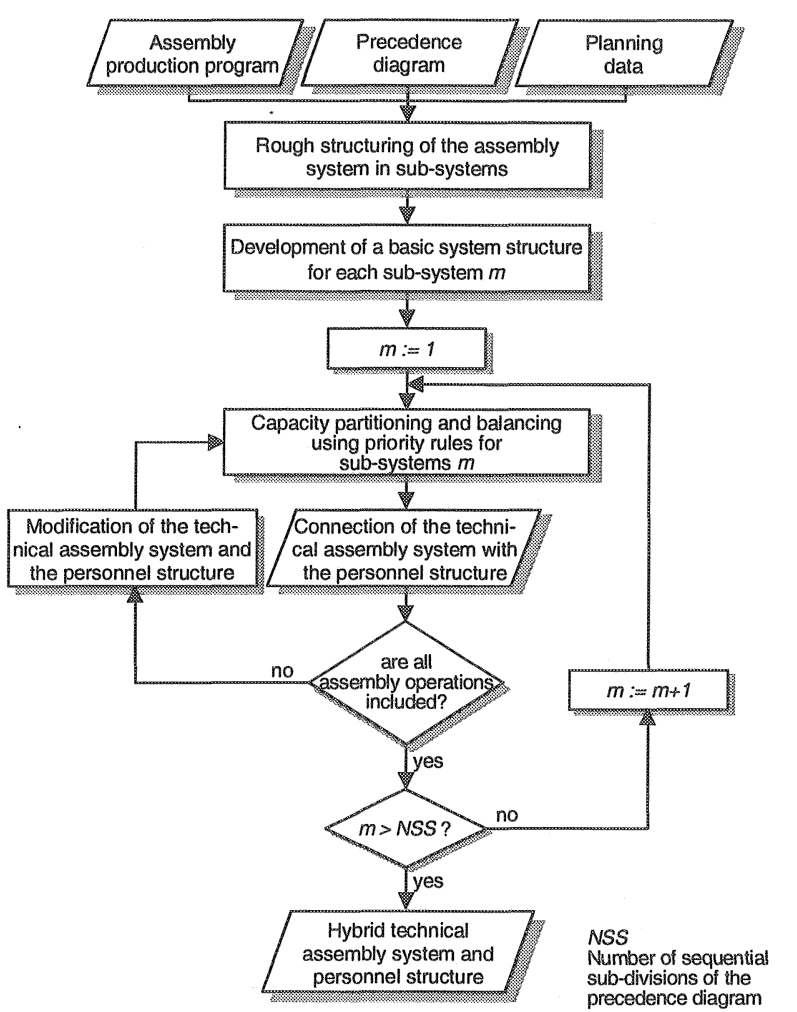

Figure 2 - General form of the planning algorithm (MÜLLER 2002, S. 117) 

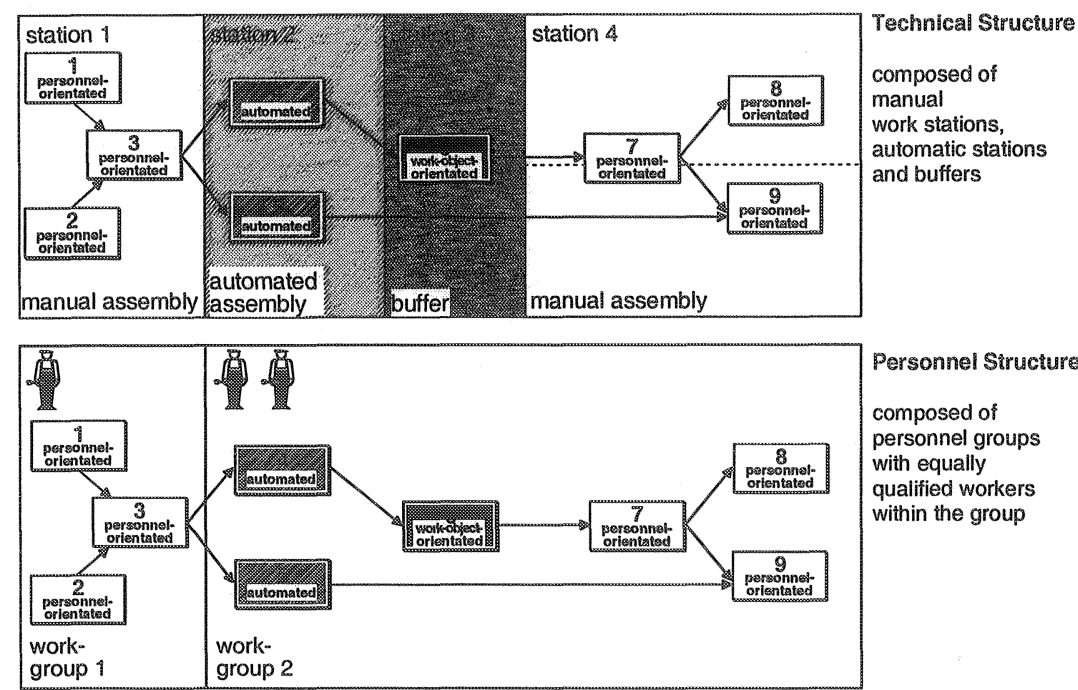

Personnel Structure composed of personnel groups with equally qualified workers qualified workers
within the group

Figure 3 - Representation of the technical and personnel structures of an assembly system

\section{PLANNING OF A GEARING MECHANISM ASSEMIBLY}

The application of the PAMELA procedure will be described using the planning case of a gearing mechanism assembly in the automobile industry. With the regarded gearing mechanisms, three main variants, which differ only slightly in their construction, can be differentiated. Based on given piece numbers a cycle time of one minute can be determined. The application of the PAMELA procedure should help discover a good planning solution.

Aside from the determination of a suitable assignment of assembly operations to stations and workers, the goal of the planning is to examine the potential for automation of the assembly operations. Therefore, the automation expenditure for each assembly operation is estimated and compared with the possible savings from the reduction of the manual execution time. In this manner those assembly operations which are particularly suited for automation can be determined.

\section{Creation of Precedence Diagrams}

At the beginning of the planning a precedence diagram for the gearing mechanism assembly is created. For this purpose so-called variant graphs are created in the PAMELA procedure for the individual gearing mechanism variants, which in this case do not differ with respect to the assembly operations or the operation sequence, rather only partially and very minimally with respect to the assembly time. The variant graphs can be summarized into a family graph which subsumes all variants of the gearing mechanisms to be assembled in the assembly system (Figure 4). An assembly precedence diagram is created for the case at hand, in which several operations are automated in accordance with the preceding economical analysis. 
The assembly precedence diagram is composed of a total of 40 assembly operations. The required planning information is inserted into each node in the precedence diagram, whereby the time values are weighted averages of the variants. Different operation times can be specified in PAMELA for the varying process participant views (worker-orientated, automatic, work-object-orientated).

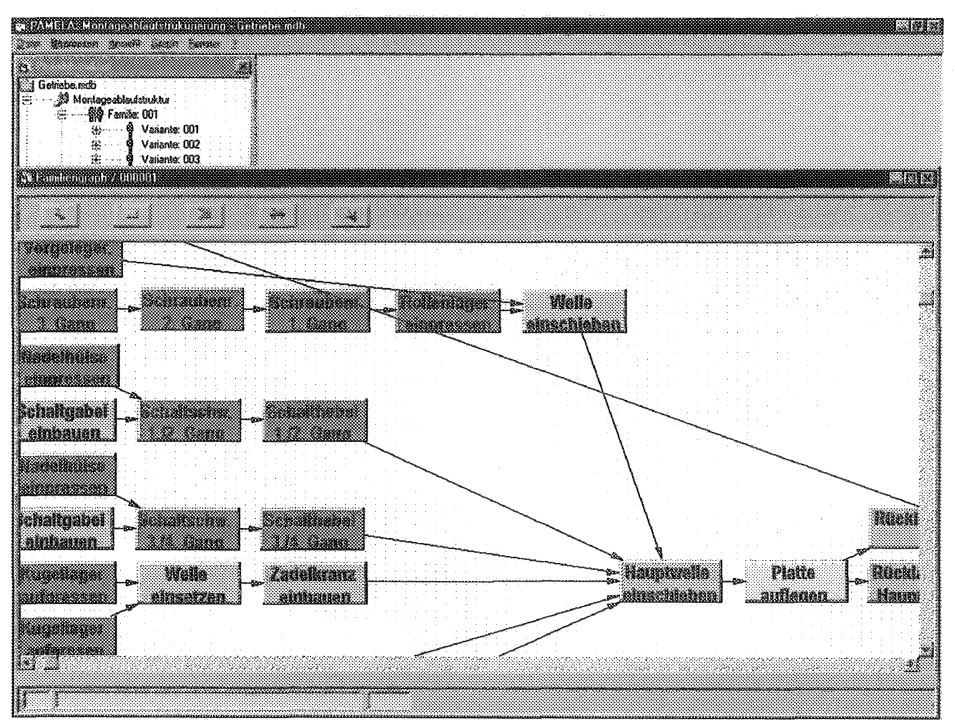

Figure 4 - Modelling of a hybrid assembly task using a precedence diagram

\section{Ballancing of the Assembly System}

Based on the precedence diagrams, a balancing of the assembly system is carried out with PAMELA. For this purpose, various planning alternatives, which differ with respect to the assignment of assembly operations and workers to stations and which can be either preferentially work type divided or order apportioned, can be developed. Apportionment of an order refers to partially parallel structures in which a worker carries out a broader assembly content upon a product, but only for a portion of the entire product quantity. 


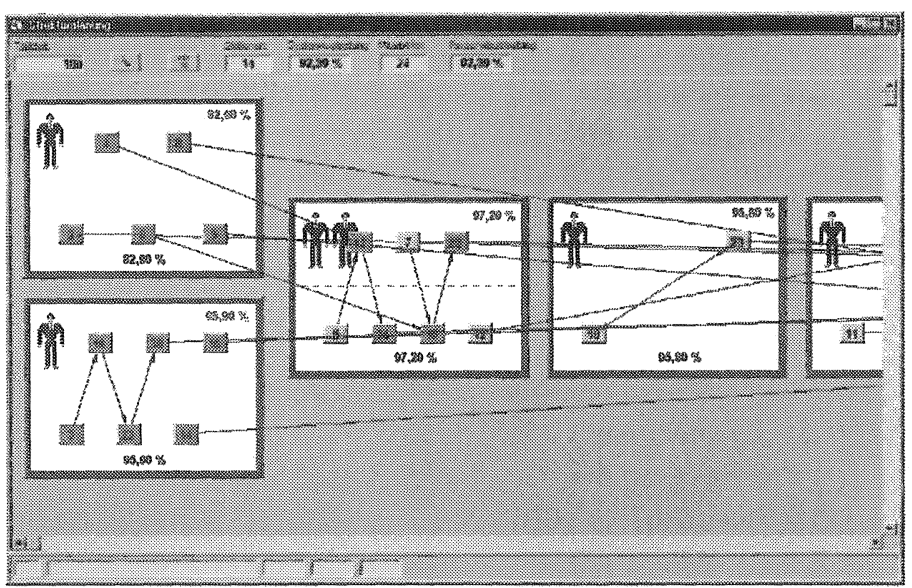

Figure 5 - Hybrid assembly system structure developed with the PAMELA procedure

The PAMELA procedure supports the planner in the balancing with, for example a graphical representation, as shown in Figure 5. For this purpose the procedure layers the technical system structure and the personnel structure, as well as the assembly operations, over one another. This representation delineates the assignment of assembly operations to stations and the sequence relationships of the assembly operations. The number of workplaces within every station is shown by horizontal lines (e.g. stations with two workplaces with one horizontal line). The assignment of workers or worker groups to one or even more stations is represented as a thick, rectangular frame. A worker can thereby be employed at several assembly stations. In addition, the graph shows the static station and worker utilization.

\section{Simulation of the Assembly System Structure}

In order to assess various planning alternatives however, a purely static consideration is not sufficient (Schiller, Muller 1997, pp. 42). This applies in particular to hybrid assembly systems and those containing a large number of variants. For this reason the planning procedure PAMELA contains an interface with the simulation procedure WITNESS (Lanner-Group 2002) for the dynamic analysis of planning alternatives. This interface allows simulation models for the planning alternatives to be generated, for the most part automatically. By using simulation, different planning alternatives can be compared.

For this purpose the assembly system structure is transferred into a simulation model in which the required workers, stations or machinery resources and buffers, along with their respective links, are modelled and displayed. Finally, the procedure PAMELA automatically starts the simulation experiment for the model created beforehand and the appropriate order programme with the simulation procedure WITNESS. In this simple manner the planner can, early in the planning stage, evaluate the planning solutions with respect to their dynamic properties and review their goal fulfilment. 
With help from the simulation of the hybrid gearing mechanism assembly system a suitable planning solution should be determined. Different alternatives, which will be simulated and compared with each other, have been developed. Some of these alternatives are characterized by preferred work type division, whereas the others are characterized by preferred order apportionment. Regarding order apportionment, parallel workplaces are planned for several purely manual assembly stations. No parallel stations are planned for automated operations in order to avoid further automation investment.

The best planning alternative is composed of 20 assembly stations with 9 station types. The system is operated with 20 workers. The results of the simulation show that this planning alternative achieves the required output, corresponding with the cycle time of one gearing mechanism per minute.

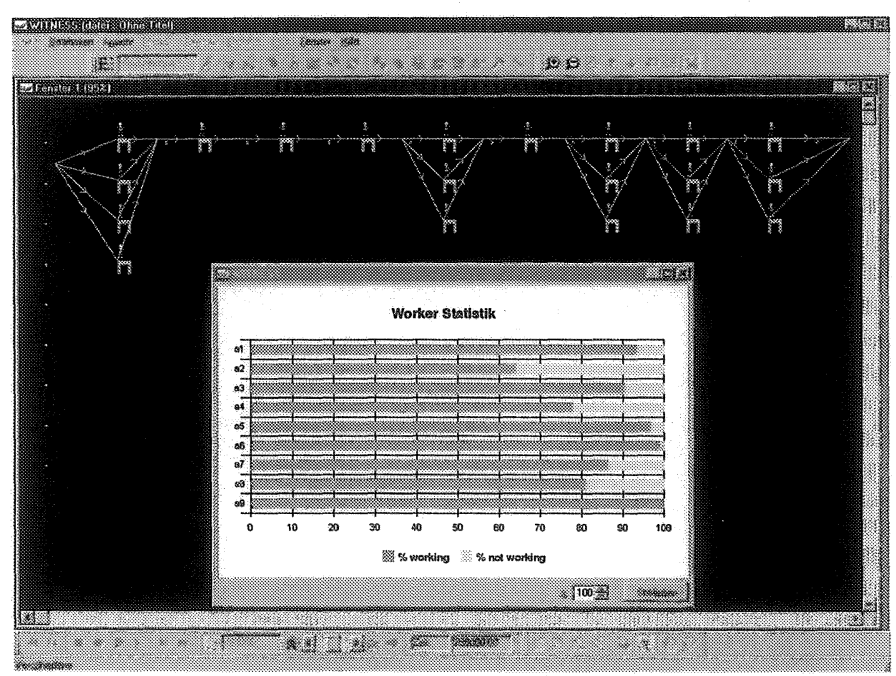

Figure 6 - Dynamic analysis of an assembly system with the procedures PAMELA and WITNESS

Figure 6 shows the respective simulation interfaces in WITNESS with a worker or worker group utilization which varies from $63.5 \%$ to $99.9 \%$. Aside from this particular Figure, the possibility to use other WITNESS statistic functions and to be presented with other relevant Figures exists. These include, for example assembly station utilization, inventories as well as buffer waiting and idle times.

\section{CONCLUSIONS}

Concluding, it can be seen that the simple handling of the PAMELA procedure provides the planner with the possibility of analysing the planning alternatives, not only in a static, but also in a dynamic manner, without broad simulation knowledge. The key Figures required for the interpretation of the simulation results are thereby prepared clearly so that possible weak points in a planning solution can be identified directly. In this manner various planning alternatives, for example with varying 
degrees of automation can be compared with each other in order to derive an appropriate hybrid system structure.

\section{REFERENCES}

[1] LANNER-GROUP: WITNESS product description. Düsseldorf: Lanner Group, 2002. http: //www.lanner.com/de/ (08.01.2002).

[2] MÜLLER, Reinhard: Planung hybrider Montagesysteme auf Basis eines mehrschichtigen Vorranggraphenkonzeptes. Aachen: Shaker Verlag, 2002 - in print. (ifab-Forschungsberichte aus dem Institut für Arbeitswissenschaft und Betriebsorganisation der Universität Karlsruhe)

[3] PRENTING, Theodore; BATTAGLIN, Robert M.: The Precedence Diagram: A Tool for Analysis in Assembly Line Balancing. In: Journal of Industrial Engineering, New York NY, 15(1964)4, pp. $209-213$.

[4] SCHILLER, Emmerich F.; MÜLLER, Reinhard: Simulationsunterstuitzte Planung der Arbeitsorganisation in manuellen Montagesystemen. Aachen: Shaker Verlag, 1997. (ifab-Forschungsberichte aus dem Institut für Arbeitswissenschaft und Betriebsorganisation der Universität Karlsruhe, Band 13)

[5] ZÜLCH, Gert; MÜLLER, Reinhard: Simulationsunterstützte Leistungsabstimmung hybrider Montagesysteme. In: Simulationstechnik. Hrsg.: PANRECK, Klaus; DÖRRSCHEIDT, Frank. Erlangen: Gruner Druck, 2001, S. 375 - 380. (=2001a)

[6] ZÜLCH, Gert; MÜLLER, Reinhard: Entwicklung von Organisationsstrukturen für die Montage. In: Ergonomie und Organisation in der Montage. Edts.: LANDAU, Kurt; LUCZAK, Holger. München: Carl Hanser Verlag, 2001, pp. 338 - 349. (=2001b) 